\title{
Testing the validity of the paddle method for the kinesthetic and visual-kinesthetic perception of inclination
}

\author{
CATINA FERESIN \\ University of Padua, Padua, Italy \\ TIZIANO AGOSTINI \\ University of Trieste, Trieste, Italy \\ and \\ NILA NEGRIN-SAVIOLO \\ University of Padua, Padua, Italy
}

\begin{abstract}
We tested the validity of the paddle method for measuring both the kinesthetic and visual-kinesthetic perception of inclination. In three conditions, subjects performed three different tasks: (1) rotating a manual paddle to a set of verbally given inclinations (blindfolded subjects), (2) rotating a manual paddle to the same set of verbally given inclinations after specific kinesthetic training (blindfolded subjects), and (3) rotating the paddle to a set of fixed visual inclinations after the kinesthetic training. The results showed a high degree of accuracy and precision in the second and third task but not in the first one. When subjects were asked to rotate a manual paddle to a set of verbally given inclinations, they used three main anchors $\left(0^{\circ}, 45^{\circ}, 90^{\circ}\right)$. Furthermore, the paddle method is biased by a kinesthetic deficiency, namely a rotational problem of the wrist that can be corrected by means of specific training.
\end{abstract}

In a celebrated work on the role of texture in the perception of inclination, Gibson (1950) used, for the first time, a visual-kinesthetic method, which he called "the method of reproduction." During that experiment, the subjects adjusted a rotatable surface (a manual paddle) with their palm until the kinesthetic impression of inclination was the same as the visual impression of inclination. ${ }^{1}$ The "method of reproduction" can also be called "the paddle method."

After Gibson (1950), many scientists used "purely visual methods." The first of these consists of a comparison line or a surface in the frontal plane used by subjects to match visually the inclination of another surface in depth (e.g., Harris, Freeman, \& Hughes, 1992; Ryan \& Gillam, 1994; van Ee \& Erkelens, 1995). The second consists of a monocular comparison between two surfaces, indicating which has the greater inclination in depth (see, e.g., Braunstein \& Payne, 1969; Freeman, 1966; Phillips, 1970). The third visual method consists of asking subjects to set the inclination of a comparison line presented after a test pattern has been removed (Epstein \& MorganPaap, 1974).

Quite recently, the visual-kinesthetic method has again been used for measuring the visual perceived inclination of a surface. This is because some researchers recognized

Correspondence should be addressed to $C$. Feresin, Dipartimento di Psicologia Generale, Università di Padova, via Venezia, 835131 Padova, Italy (e-mail:paola@psicoserver.univ.trieste.it). that this method does not introduce other visual stimuli that might have an effect upon the measurement (Carrozzo \& Lacquaniti, 1994; Howard \& Kaneko, 1994; Ohmi, 1993; Proffitt, Bhalla, Gossweiler, \& Midgett, 1995). For example, the intersensory method cannot be affected by visual aftereffects from the test pattern, or by the depth contrast effect.

Howard and Kaneko (1994) and Proffitt et al. (1995) addressed the validity of the paddle method. Howard and Kaneko found an underestimation of a large range of inclinations of visually perceived surfaces having a full range of binocular and monocular depth cues. To calibrate their unseen paddle before starting a set of experiments, they asked the subjects to match the inclination of the paddle to a very big cardboard surface. This surface was covered with random dots and was inclined at $10^{\circ}$ intervals between $+80^{\circ}$ and $-80^{\circ}$. They used a third-order polynomial function, which best fit the underestimation they found, as a baseline for comparing the results of the calibration with the results of the experimental conditions.

Proffitt et al. (1995) used many psychophysical methods to measure the geographical inclination of hills. Their subjects gave verbal estimates of the inclination of a set of hills, adjusted a representation of the cross section of those hills, and, finally, used the visual-kinesthetic method, setting a tilt board with their hands unseen. Proffitt et al. found a small underestimation of the visually perceived inclination of a set of hills when using the tilt board and, overall, this measure was different from the 
actual inclinations of the hills. In their paper, Proffitt et al. measured the internal consistency among the three already mentioned psychophysical methods they used. They compared the kinesthetic responses the subjects made by looking at some inclined hills with the kinesthetic responses that the same subjects made in response to an almost equivalent set of verbally given angles. The subjects displayed an internal consistency for both the verbal-kinesthetic and the visual-kinesthetic measures.

We decided to approach the problem of the validity of the paddle method in a different way than did Howard and Kaneko (1994) and Proffitt et al. (1995). If a person is observed rotating his/her wrist, it is quite clear that there is one main motor deficiency: The wrist cannot rotate completely to achieve the vertical position when the forearm is orthogonal with respect to the arm (Figure 1A). To avoid this rotational problem close to the vertical, the person has to lift his/her forearm up with respect to the arm and incline the arm and the shoulder by a small amount (Figure 1B). However, close to the horizontal, the subject's wrist may have an impediment in its rotation due to the inclined position of the forearm with respect to the arm (Figure 2B). Because of this, the subject cannot really achieve a correct position of the wrist and the forearm at the horizontal position (Figure 1 A). As a consequence of this possibie motor problem around the vertical, it is best for the person to choose a position for the forearm and the wrist that allows him/her to freely rotate the paddle from the vertical to the horizontal. This position results in a compromise between the best position for
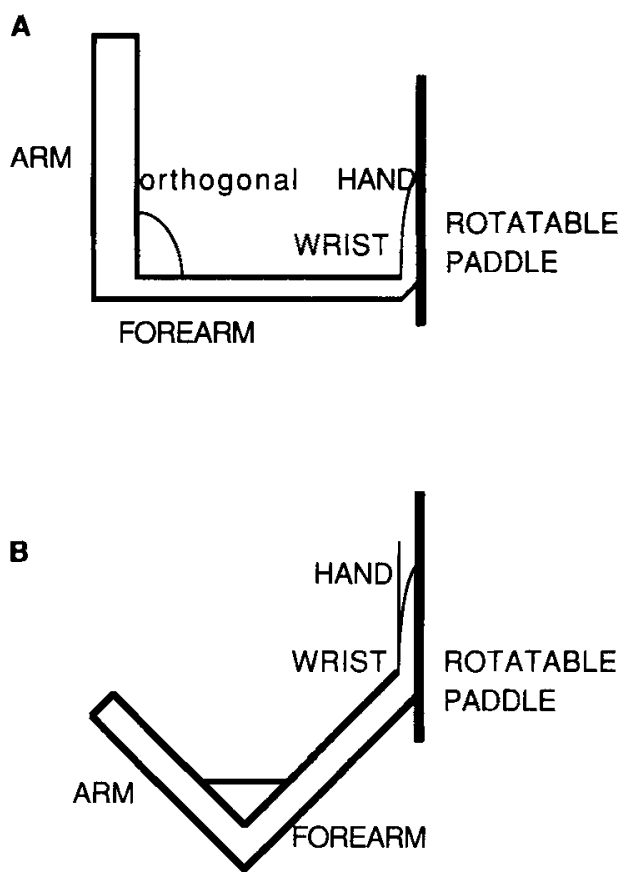

Figure 1. (A) Kinesthetic deficiency of the wrist at the vertical position when the forearm is orthogonal with respect to the arm. (B) To solve the kinesthetic deficiency at the vertical position, the observer has to lift up his/her forearm and incline the arm.
A

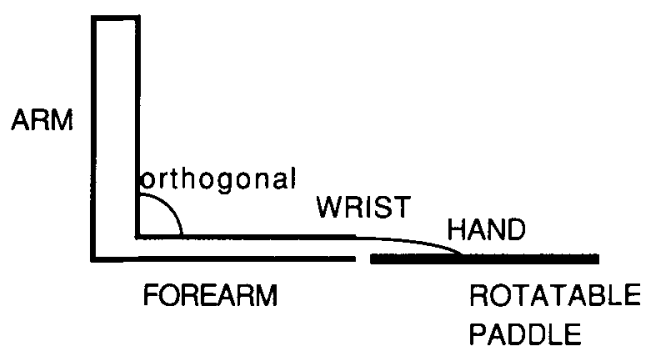

B

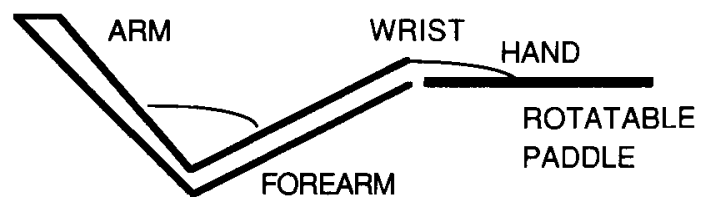

Figure 2. (A) Correct position of the wrist and the forearm at the horizontal position. (B) Uncomfortable position of the wrist to achieve the horizontal position due to the inclination of the forearm and arm.

rotating the wrist to the vertical and the best position for rotating the wrist to the horizontal.

Because of this compromise, we suppose that when a person is asked to kinesthetically set a paddle to the vertical position $\left(0^{\circ}\right)$, he/she will rotate the wrist more than $0^{\circ}$, stopping the setting forward and then overestimating the perceived inclination (Figure 3A). On the contrary, when a person is asked to kinesthetically set a paddle to the horizontal $\left(90^{\circ}\right)$, we suppose that he/she will rotate the wrist less than $90^{\circ}$, stopping the setting backward and then underestimating the perceived inclination (Figure 3B).

Furthermore, we think that it is a reasonable strategy for subjects to find and use spontaneously some kind of kinesthetic anchor. This is because we know from the literature that the vertical and the horizontal are the main visual and haptic orientational anchors or norms (e.g., Cecala \& Garner, 1986; Gentaz \& Hatwell, 1995; Howard, 1982). When people are asked to judge the visual and the haptic inclination of a surface or a rod, they use the horizontal and the vertical plane as a frame of reference. So, why should it be different in the kinesthetic domain?

The first aim of our research was to investigate the purely kinesthetic perception of a range of inclined surfaces to test whether the paddle method is biased in some way by motor problems or strategies used by the subjects. The second purpose was to measure the perception of the visual inclination of a line by means of the paddle kinesthetic method. If the paddle method is influenced by motor problems, as we suppose, it should be possible 


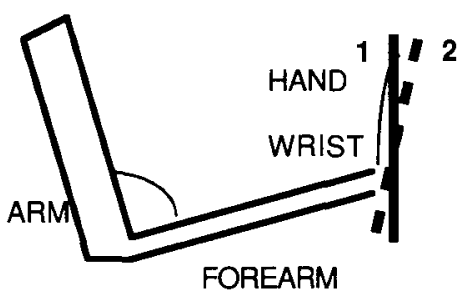

A

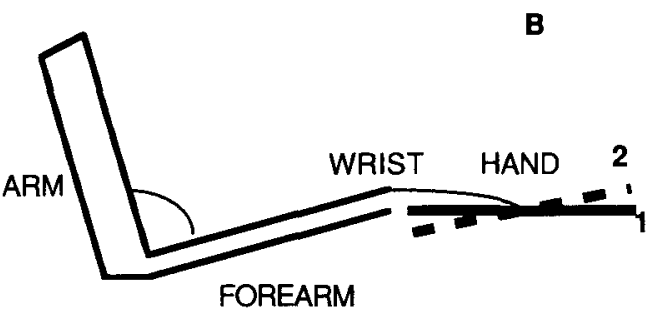

Figure 3. (A1) Correct inclination of the paddle at the vertical: uncomfortable inclination of the wrist. (A2) Overestimated inclination of the paddle at the vertical: comfortable inclination of the wrist. (B1) Correct inclination of the paddle at the horizontal: uncomfortable inclination of the wrist. (B2) Underestimated inclination of the paddle at the horizontal: comfortable inclination of the wrist.

to compensate for such motor biases with an appropriate kinesthetic training. When the motor biases are avoided, we can use the paddle method to test the perception of the visual inclination of a surface or a line. In this way it is possible to separate the kinesthetic outcome from the visual outcome.

\section{PRELIMINARY STUDY}

During a preliminary study, we observed that, while doing the kinesthetic task of rotating the manual paddle to a set of 11 inclinations going from the vertical $\left(0^{\circ}\right)$ to the horizontal $\left(90^{\circ}\right)$, subjects were limited in the extent of rotation of the wrist. The subject's wrist could rotate completely to achieve the vertical or horizontal position only by forcing it. But since the subjects were not asked to do that, they stopped their adjustment of the vertical and the horizontal position whenever they started to feel the impediment of rotating the wrist.

We also noticed that the subjects used three main kinesthetic anchors to refer their paddle adjustment: $0^{\circ}$ (gravitational vertical), $45^{\circ}$, and $90^{\circ}$ (horizontal). The subjects' use of the vertical and the horizontal anchors is logical for the reason explained in the introduction; but the subjects also used $45^{\circ}$ as an anchor. This anchor was derived in that it was achieved by the subjects as the middle position between the vertical and the horizontal. For example, when the subjects had to set the paddle to $45^{\circ}$, they first started from the vertical, then they went to the horizontal, and finally they found the middle position corresponding to $45^{\circ}$. When the subjects were asked to set the paddle to $40^{\circ}$ or $50^{\circ}$, they first looked for $0^{\circ}$ and for $90^{\circ}$, then they reached $45^{\circ}$, and finally went from it to reach $40^{\circ}$ or $50^{\circ}$.

At the end of the preliminary study, we asked the subjects if they had used any anchor as a reference point. All of them told us that they were using the three anchors we had observed while watching them doing the kinesthetic task.

\section{EXPERIMENT}

In two conditions, we measured the accuracy and precision (Ono, 1993) of adjusting the inclination of a manual paddle to a set of verbally given angles. In a third condition, we measured the accuracy and precision of adjusting the inclination of the same manual paddle to a set of visually given inclinations.

The measure of accuracy was the mean of the point of subjective equality (PSE) compared with the point of objective equality (POE). The measure of precision was the standard error.

The task performed by the subjects in the first and second conditions was a purely kinesthetic task in that the inclination of the manual paddle was not compared with a visual surface and the observer was blindfolded. We also investigated the subject's possible strategies in performing the task. The task performed by the subjects in the third condition was a visual-kinesthetic task in that the inclination of the manual paddle was compared with a set of inclinations of a luminous line.

\section{Method}

\section{Subjects}

Eighteen subjects took part in the experiment-6 for each condition. They were undergraduate and graduate psychology students of the University of Trieste. All were volunteers and naive regarding the aim of this work.

\section{Apparatus}

A cubic chamber, $150 \mathrm{~cm}$ long on each side, was placed in the middle of the laboratory. A rotatable paddle $(24 \mathrm{~cm}$ long $\times 16 \mathrm{~cm}$ wide $\times 3 \mathrm{~cm}$ thick) was connected to the frame of the chamber by means of a small table. The paddle was connected to an electronic protractor (Emaco Angle Star protractor system, Montreal, Quebec, Canada). During the third condition, the chamber was illuminated by two projectors placed outside the right and the left sides of the chamber itself. A thick luminous line $(35 \mathrm{~cm}$ long $\times 1.5 \mathrm{~cm}$ wide) was mounted on a wooden rotatable bar and fixed to the cubic chamber in front of the subject at a viewing distance of $150 \mathrm{~cm}$. The center of the line was at eye level (Figure 4).

\section{Procedure}

Condition 1. The blindfolded subject was placed at the center of the chamber and seated on an adjustable chair. After the subject had chosen the most comfortable position, which remained constant during all the trials, the experimenter taped the hand to the paddle. The tape did not interfere with the free movement of the wrist. The taping was done to prevent movements of the fingers that could have influenced the inclination response of the wrist. The paddle was not a weight on the hand, and of course there was 


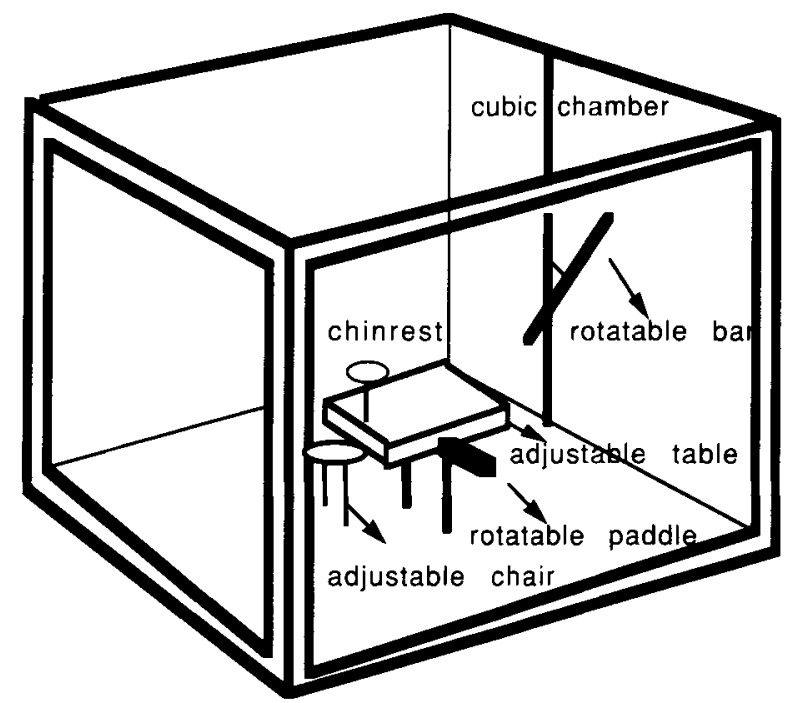

Figure 4. Cubic chamber.

no change of one of the limb's eigenvectors (Pagano \& Turvey, 1995).

The subject was asked to set the manual paddle to a set of verbally given inclinations relative to gravity. On each trial, the subject stopped when the kinesthetic inclination of the wrist felt the same as the verbal inclination.

Before performing Condition 1, the subjects were tested for their understanding of the entire set of angles. Indeed, they were asked to reproduce the entire set of angles by rotating the wrist.

Eleven verbal angles went from the gravitational vertical $\left(0^{\circ}\right)$ to the horizontal $\left(90^{\circ}\right)$ in steps of $10^{\circ}\left(45^{\circ}\right.$ included). The 11 settings were performed in random order, and every setting was repeated in random order five times, yielding a total of 55 trials for each subject. Subjects started all their settings from a random inclination of the paddle.

Condition 2. The aim of Condition 2 was to investigate the subjects' performance after we had trained them to use the three anchors strategy and to overcome the rotational deficiency of the wrist that we had noticed during the pilot study. This task, like the one in the first condition, was a purely kinesthetic task in that the inclination of the manual paddle was not compared with any visual surface inclination and the subject was blindfolded.

The procedure followed by the subjects in the second condition was the same as the procedure of Condition 1 except for training. Indeed, we trained the subjects to use the three main anchors $\left(0^{\circ}\right.$, $45^{\circ}$, and $90^{\circ}$ ) that had been used spontaneously by the group of during in the pilot study. First, we taught them to rely on the vertical anchor $\left(0^{\circ}\right)$ when they had to set the paddle to inclinations from $10^{\circ}$ to $30^{\circ}$ verbally indicated, to rely on the $45^{\circ}$ anchor for inclinations from $40^{\circ}$ to $50^{\circ}$, and finally to rely on the horizontal anchor $\left(90^{\circ}\right)$ for inclinations from $60^{\circ}$ to $80^{\circ}$.

The training method we used was the basic one of demonstration and feedback for the whole set of inclinations. Furthermore, we told the subjects about the rotational deficiency, and we trained them not to stop their adjustment when they started to feel the impediment in rotating the wrist. We expected an increase of accuracy and precision as a result of this training compared with the accuracy and precision obtained in Condition 1.

Condition 3. The purpose of Condition 3 was to test subjects visually perceived inclination of a line stimulus characterized by most of the monocular and binocular depth cues. All the external frames were visible; indeed, the edges of the cubic chamber were used by the subject as a frame of reference to incline the luminous line. This line was thick enough for linear perspective. We measured this visually perceived inclination by means of the paddle.

In this final condition, we trained the subjects to pay attention to the motor deficiency of the wrist and to use the three anchors, as we had done in Condition 2. This task was a visual-kinesthetic task in that the inclination of the manual paddle was compared with inclinations of the luminous line.

The independent variable was the set of visual inclinations of the line - that is, the POE - whereas the dependent variable was the manual adjustment of the inclination of the paddle - that is, the PSE. The levels of the independent variable varied in steps of $10^{\circ}$ $\left(45^{\circ}\right.$ included) between gravitational vertical $\left(0^{\circ}\right)$ and $80^{\circ}$. We did not use the $90^{\circ}$ inclination (horizontal) because it was perceived by the subject as a point in space and not as an inclination. The kinesthetic training was the same as in Condition 2.

The subject was placed at the center of the cubic chamber, as in Conditions 1 and 2, resting his/her head on a chinrest, and was asked to rotate the manual paddle to each of a set of inclinations of the luminous line. During this condition, the subject stopped each trial when the kinesthetic inclination of the paddle was judged to be the same as the visual inclination of the luminous line.

The line was inclined by the experimenter to 1 of 10 angles presented in random order. Each angle was repeated in random order five times, yielding 50 total trials for every subject.

\section{Results}

\section{Condition 1}

As Figure 5 shows, the 6 subjects overestimated (forward direction) the kinesthetic inclinations relative to the verbal angle from $0^{\circ}$ to $20^{\circ}$; settings were very accurate but not very precise at $30^{\circ}$, quite accurate and precise (small standard errors) at $40^{\circ}$, and very accurate and precise at $50^{\circ}$. Finally, the subjects underestimated (backward direction) the kinesthetic inclinations relative to the verbal angle from $60^{\circ}$ to $90^{\circ}$.

When the verbal angle was close to the gravitational vertical (from $10^{\circ}$ to $30^{\circ}$ ), subjects always started their settings from the vertical. This means that if they tended to slightly overestimate the vertical itself, stopping their

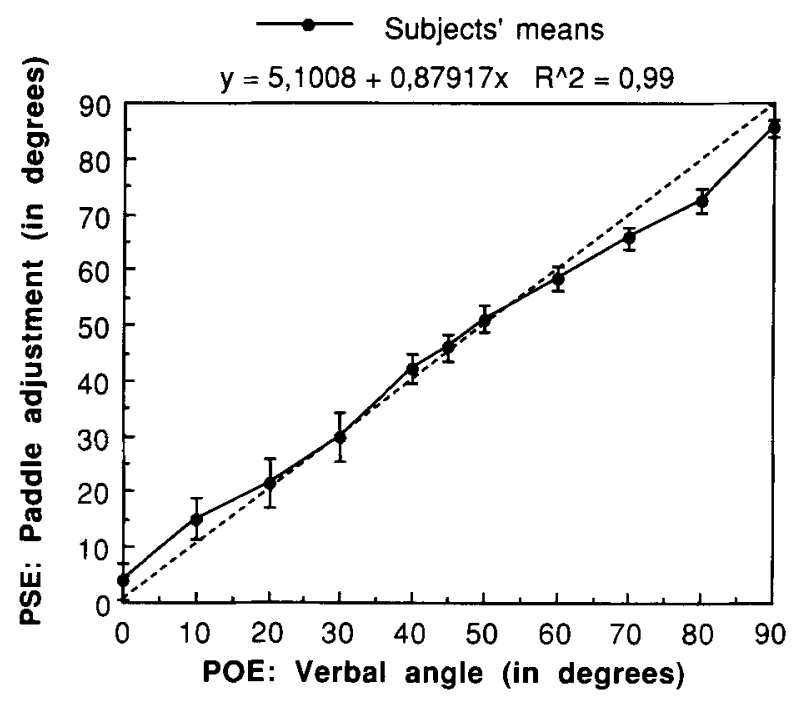

Figure 5. Mean results of Condition 1: kinesthetic task. 
setting forward, they carried this initial overestimation with them and also showed this bias for angles close to the vertical.

It was the same for the horizontal. Indeed, the subjects tended to underestimate the horizontal, stopping their setting backward, and carried this underestimation with them, also showing it for angles close to $90^{\circ}$ (from $60^{\circ}$ to $80^{\circ}$ ).

We divided the distribution into two halves - from $0^{\circ}$ to $40^{\circ}$ and from $50^{\circ}$ to $90^{\circ}$, excluding the $45^{\circ}$ since it is the inversion point between positive and negative PSE. Then we subtracted the paddle settings from the verbally defined values; finally, we analyzed the two distributions by using a one-group $t$ test. The statistical analysis showed a significant difference between the paddle settings and the verbal values for the $0^{\circ}-40^{\circ}$ distribution $[t(4)=-2.8$, $p<.05]$ and a tendency to significance in the opposite direction for the $50^{\circ}-90^{\circ}$ distribution $[t(4)=2.4, p<.07]$.

\section{Condition 2}

Figure 6 shows mean responses for each verbal angle. The 6 subjects were more precise than in Condition 1, as the small standard errors show. Furthermore, there was no over- or underestimation of the perceived kinesthetic inclination. Indeed, the subjects showed high accuracy from $0^{\circ}$ to $90^{\circ}$.

We analyzed the data in the same way as in Condition 1. A one-group $t$ test showed no significant difference between the paddle settings and the verbal values for either the $0^{\circ}-40^{\circ}$ distribution $[t(4)=-1.50, p<.20]$ or the $50^{\circ}-90^{\circ}$ distribution $[t(4)=-0.20, p<.85]$.

\section{Condition 3}

Figure 7 shows the mean responses for each visual inclination. The 6 subjects were very accurate and precise when they kinesthetically adjusted the paddle to the 10 different visual inclinations.

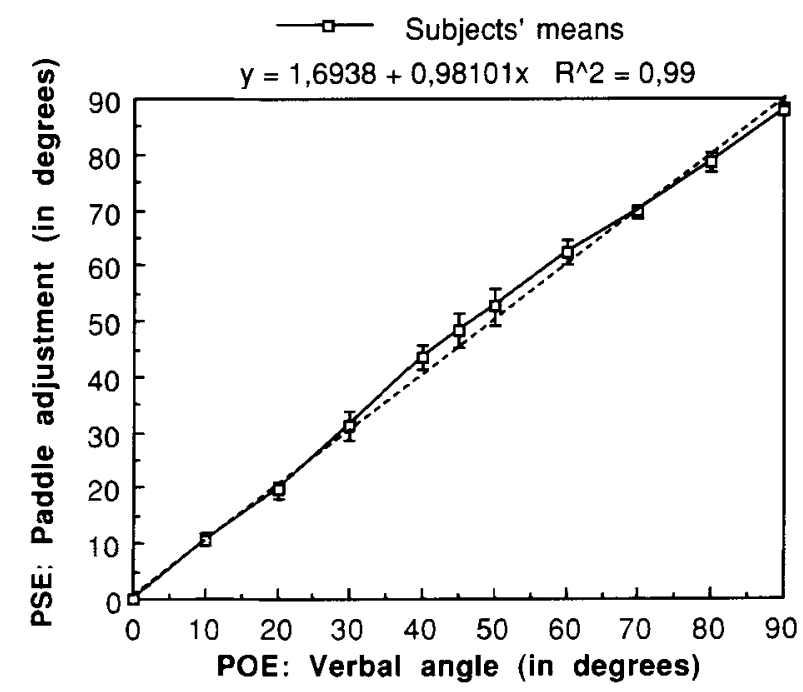

Figure 6. Mean results of Condition 2: training + kinesthetic task.

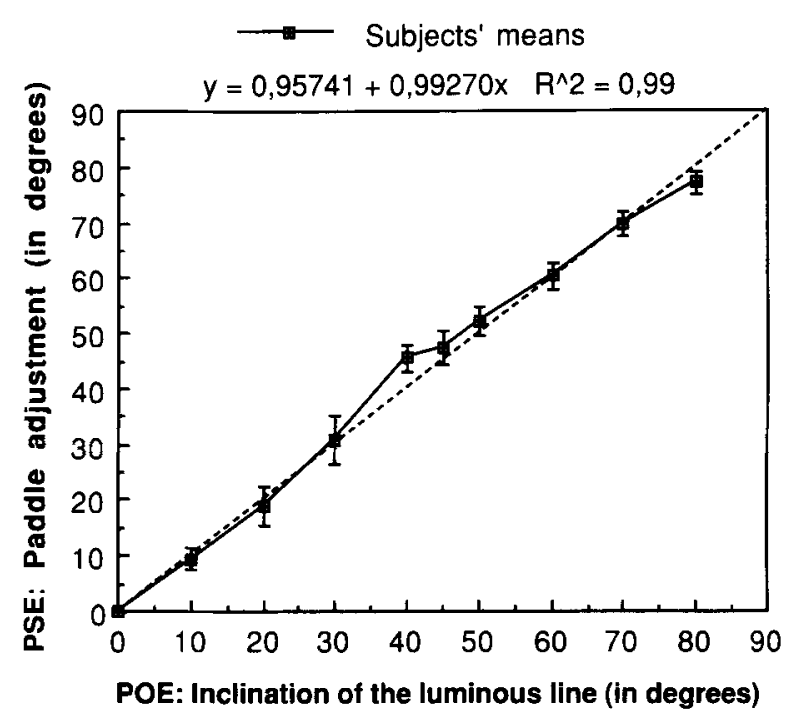

Figure 7. Mean results of Condition 3: training + visualkinesthetic task.

A one-group $t$ test showed no significant difference between the paddle settings and the visual inclinations for either the $0^{\circ}-40^{\circ}$ distribution $[t(4)=-0.71, p<.51]$ or the $50^{\circ}-80^{\circ}$ distribution $[t(4)=0.05, p<.96]$.

\section{DISCUSSION}

In the first condition, in which the subjects were not trained to use the paddle, we found an underestimation of the perceived kinesthetic inclination when the tested angles were close to the horizontal, an overestimation when they were close to the gravitational vertical, and a quite good level of accuracy and precision around $45^{\circ}$. In the second condition, in which subjects were trained to use three anchors (spontaneously used in the pilot study and in the first condition) and received feedback to compensate for the wrist rotational problem, we observed a good level of accuracy and precision of the responses without any under- or overestimation. In the third condition, the subjects had the same kinesthetic training as in Condition 2 before performing a visual-kinesthetic task. The results of this final condition showed a high degree of accuracy and precision of the responses.

The main point emerging from this research is that the use of the paddle method presents a wrist rotational bias due to the position of the subject's forearm while he/she is performing a kinesthetic task. However, the underestimation and overestimation found in the first condition nearly disappears when the rotational bias is compensated for by giving the subject kinesthetic feedback concerning the veridical inclination of the paddle.

We emphasize that, when the subjects were asked to perform a kinesthetic task by rotating a manual paddle, they spontaneously used three anchors - that is, the perceived $0^{\circ}, 45^{\circ}$, and $90^{\circ}$. We suppose that training the sub- 
jects to consciously use these anchors reduced data variability by preventing the use of uncontrolled strategies. Furthermore, we suppose that the kinesthetic outcome could be affected by the use of strategies different from those we found in our first condition, and we hope to investigate them in a future experiment. Most important, we think that kinesthetic variables such as the effect of wrist and forearm position should be taken into account when the paddle method is used.

Our results show that in a visual-kinesthetic task, an inclined line is matched correctly when subjects are previously trained in the kinesthetic domain. This suggests that use of the intersensory method without training could measure a motor problem and the presence of heterogeneous strategies rather than a visual misperception. In conclusion, we think the intersensory paddle method is a valid one but only when precautions are taken by training the subjects to overcome the rotational problem of the wrist and by verifying their strategies.

\section{REFERENCES}

Braunstein, M. L., \& Payne, J. W. (1969). Perspective and form ratio as determinants of relative slant judgments. Journal of Experimental Psychology, 81, 584-590.

Carrozzo, M., \& LacQuaniti, F. (1994). A hybrid frame of reference for visuo-manual coordination. NeuroReport, 5, 453-456.

CECAlA, A. J., \& GARNER, W. R. (1986). Internal frame of reference as a determinant of the oblique effect. Journal of Experimental Psychology: Human Perception \& Performance, 12, 314-323.

EPSTEIN, W., \& MORGAN-PAAP, C. L. (1974). Aftereffect of inspection of a perspectival stimulus for slant depth: A new normalization effect. Perception \& Psychophysics, 16, 299-302.

Freeman, R. B. (1966). Effect of size on visual slant. Journal of Experimental Psychology, 71, 96-103.

GentAZ, E., \& HATwell, Y. (1995). The haptic "oblique effect" in children's and adults' perception of orientation. Perception, 24, 631-646.
Gibson, J. J. (1950). The perception of visual surfaces. American Journal of Psychology, 63, 367-384.

Harris, M., Freeman, T., \& Hughes, J. (1992). Retinal speed gradient and the perception of surface slant. Vision Research, 32, 587-590.

Howard, I. P. (1982). Human visual orientation. New York: Wiley.

Howard, I. P., \& KANeko, H. (1994). Relative shear disparities and the perception of surface inclination. Vision Research, 34, 2505-2517.

KLATZKY, R. L., \& Lederman, S. J. (1995). Identifying objects from a haptic glance. Perception \& Psychophysics, 57, $1111-1123$.

OHMI, M. (1993). Depth perception of wide-angle surface [Abstracts of the Sixteenth European Conference on Visual Perception]. Perception, 22 (Suppl.), 114.

ONo, H. (1993). Precision and accuracy in perception [Educational Computer Program Package]. Santa Barbara, CA: Intellimation.

Pagano, C. P., \& Turvey, M. T. (1995). The inertia tensor as a basis for the perception of limb orientation. Journal of Experimental Psychology: Human Perception \& Performance, 21, 1070-1087.

PHILliPS, R. J. (1970). Stationary visual texture and the estimation of slant angle. Quarterly Journal of Experimental Psychology, 22, 389. 397.

Proffitt, D. R., Bhalla, M., Gossweiler, R., \& Midgett, J. (1995). Perceiving geographical slant. Psychonomic Bulletin \& Review, 2 , 409.428.

Ryan, C., \& Gillam, B. (1994). Cue conflict and stereoscopic surface slant about horizontal and vertical axes. Perception, 23, 645-658.

van Ee, R., \& ERKelens, C. J. (1995). Binocular perception of slant about oblique axes relative to a visual frame of reference. Perception, 24, 299-314.

\section{NOTE}

1. Similar to Klatzky and Lederman (1995), we define kinesthesis as perceptual information coming from muscles, tendons, and joints, whereas cutaneous perceptual information derives from impressions coming from somatosensory inputs of the skin. Kinesthetic and cutaneous perceptual information are both parts of the haptic system.

(Manuscript received January 13, 1997; revision accepted for publication May 14, 1997.) 